\title{
Extinction of taste aversion does not eliminate taste-mediated aversion to visual cues: Replicating Lett (1984)
}

\author{
JOSEPH J. FRANCHINA, DAVID J. WRIGHT, ROBERT F. SMITH, CHERYL Y. PENN, \\ and ELIZABETH SOEKEN \\ Virginia Polytechnic Institute and State University, Blacksburg, Virginia
}

\begin{abstract}
In this experiment, we evaluated whether extinction of taste aversion following conditioning with a compound CS of visual and taste cues would attenuate taste-mediated aversion to visual cues. Sixty chicks (Gallus domesticus) received a pairing of a compound CS, red-colored water or red-colored vinegar, with a toxin and then extinction procedures with clear vinegar. Testing with red water showed reliable evidence of potentiation to visual cues in the red vinegar conditioning groups, as measured by latency to start drinking and intake performance. Taste-aversion extinction groups $(n=30)$, tested with clear vinegar, showed no reliable evidence of taste aversion. The results contradicted the within-compound hypothesis of taste-mediated potentiation and replicated Lett (1984).
\end{abstract}

In taste-mediated potentiation of aversion to visual cues, pairing a compound conditioned stimulus (CS) of visual and taste cues with a toxin yields greater aversion to visual cues than pairing the toxin with visual cues alone (Lett, 1980). The within-compound association (WCA) view (Durlach \& Rescorla, 1980) attributes potentiation to a summation of associations that occurs between stimulus elements and between each element and toxicosis. The synergistic compound (SC) view (e.g., Rusiniak, Hankins, Garcia, \& Brett, 1979) proposes that taste enhances the associability between visual cues and toxicosis because taste cues "gate" visual cues into the gastrointestinal system, which is shared by taste and gastric events that are presumably highly associable. No transfer of aversion occurs from taste to visual cues. If potentiation depends on a summation of associations, as the WCA hypothesis suggests, intervening taste-extinction procedures between conditioning and testing should subtract from that summation and reduce the magnitude of potentiation. Lett (1984) followed this logic and reported that extinction of taste aversion left potentiation to visual cues intact. This finding contradicted the WCA view and, by implication, supported the SC view.

Lett (1984) measured potentiation effects by examining the intake of colored water. In that study, water was a familiar solution from both the preexperimental period and during recuperation after toxicosis. Lett proposed that if detection of the familiar taste of water occurred after the start of drinking in testing, the presence of familiar taste cues may have curtailed the display of aversion to

Address correspondence to J. J. Franchina, Department of Psychology, Virginia Polytechnic Institute and State University, Blacksburg, VA 24061-0436. visual cues. Thus, an intake measure of potentiation may underestimate the effect size.

To circumvent this possibility and replicate Lett's (1984) findings, in the present experiment, latency of approach to visual cues prior to onset of drinking was measured. According to a two-stage model of ingestive behavior (e.g., Brett, Hankins, \& Garcia, 1976), distal information from visual cues of ingesta elicits approach and the onset of ingestion. If visual cues acquire aversive properties, approach latencies should be longer when the strength of aversion is greater. If the intervention of procedures that extinguish taste aversion alters potentiation to visual cues, approach latencies should be faster than those following the absence of intervening extinction (Lett, 1984).

\section{METHOD}

Subjects, Design, and Procedure

Ninety White Leghorn chicks from the Center for Poultry Research at Virginia Polytechnic Institute and State University were housed in groups of 6 until they were 8-9 days old. Sixteen hours before the start of the experiment, the chicks were placed individually into cages (interior dimensions: $24.4 \times 12.0 \times 12.0 \mathrm{~cm}$ ) with food available ad lib, but without fluids.

Sixty chicks were randomly assigned to cells of a $2 \times 2$ design: two compound CS conditions (red-colored tap water or red-colored vinegar) and two intervention procedures (clear water access/no extinction or clear vinegar access/taste-aversion extinction). These chicks were tested for potentiation effects to red-colored water. The remaining $\mathbf{3 0}$ chicks received conditioning with red water or red vinegar, followed by extinction procedures and testing with clear vinegar. If the conditioning groups did not differ on vinegar intake in testing, this would indicate the effectiveness of extinction procedures on aversion to vinegar in the red vinegar CS group.

The experiment lasted 6 days. On Day 1, at 1600 h, each chick received $10 \mathrm{~min}$ in the experimental chamber (interior dimensions: $41.6 \times 24.0 \times 17.8 \mathrm{~cm}$ ). On Day 2 , at $0830 \mathrm{~h}$, each chick received four bill dips into the appropriate CS solution, followed immediately by an intraperitoneal injection of $.4 \mathrm{M} \mathrm{LiCl}$ at $1.0 \%$ body weight. For recuper- 
ation from toxicosis, all the chicks received ad-lib access to food and clear water on Day 2 from 1400 to $1600 \mathrm{~h}$, and on Day 3 from 0830 to $1000 \mathrm{~h}$ and 1630 to $1800 \mathrm{~h}$. Otherwise, all fluids were absent. Extinction procedures started on Day 4 at $0830 \mathrm{~h}$. To assess the strength of taste aversion to clear vinegar at the start of extinction, half of the chicks in each compound CS group received 4-min access to clear vinegar and the other half received clear water. After intake was measured, the appropriate solution remained available ad lib until $1300 \mathrm{~h}$ on Day 5 . All fluids were then removed until $0830 \mathrm{~h}$ on Day 6. On Day 6, the chicks received a 4-min test trial with red-colored water $(n=60)$ to measure potentiation, or a test with clear vinegar $(n=30)$ to measure taste aversion to vinegar. For a test trial, each chick was placed at the rear of the experimental chamber, facing a clear glass jar that was $6.4 \mathrm{~cm}$ in diameter, $5.8 \mathrm{~cm}$ deep, and $4.5 \mathrm{~cm}$ away. The jar contained $55-60 \mathrm{cc}$ of solution. Release of the chick started a timer that measured latency to the first bill dip (BD) and latency to complete nine more bill dips (to the nearest $.1 \mathrm{sec}$ ). A bill dip was defined as a chick's breaking the surface of the solution with its bill, throwing its head back, and palpitating its throat. At the end of the test trial, intake was measured as the difference in the weight of the jar (to the nearest $.1 \mathrm{~g}$ ) from preto posttest. For red water, $10 \mathrm{ml}$ of red food coloring was mixed with $1,000 \mathrm{ml}$ of tap water for a $1.0 \%$ solution. For red vinegar, $20 \mathrm{ml}$ of white vinegar was mixed with $1,000 \mathrm{ml}$ of red water for a $2.0 \%$ vinegar solution.

\section{RESULTS}

Figure 1 presents mean latency to start drinking (BD1) red water and to make nine more bill dips (BD2-10) for the groups conditioned with a compound CS of red water (RW) or red vinegar (RV) and extinguished (EXT) with clear vinegar or not. The chicks in the RV/CS condition showed reliably longer latencies to start drinking than did those in the RW/CS condition for both of the extinction procedures, with no reliable differences between them [CS, $F(1,56)=18.93, p<.001$; EXT, $F<1.0]$. These data provided reliable evidence of taste-mediated potentiation to red visual cues and showed that potentiation was nondifferential for the extinction procedures. Similar results appeared in the latency data for BD 2-10 [CS, $F(1,56)=$ $10.74, p<.001]$. There were no reliable interactions in the analysis of either data set.

The intake measure corroborated the latency data. Mean intakes (in grams) of red water were 1.5 and .4 for the $\mathrm{RV} / \mathrm{CS}$ groups, and 3.9 and 3.0 for the RW/CS groups, under the no-extinction and extinction procedures, respectively $[\mathrm{CS}, F(1,56)=14.83, p<.001 ; \mathrm{EXT}, F<1.0]$.

To demonstrate evidence of taste aversion to vinegar and the subsequent extinction of vinegar aversion, the $\mathrm{RW} / \mathrm{CS}$ and RV/CS groups were compared with regard to intake of vinegar and water at the start of extinction procedures, and then the aversion-extinction groups were compared with regard to vinegar intake in testing. At the start of extinction, mean intakes of clear vinegar for the $\mathrm{RW} / \mathrm{CS}$ and $\mathrm{RV} / \mathrm{CS}$ groups were 5.0 and $2.3 \mathrm{~g}$, respectively $[t(28)=8.42, p<.001]$. Mean intakes of clear water were 6.2 and $5.4 \mathrm{~g}$, respectively $(t<1.0)$. Thus, at the start of extinction, the RW/CS and RV/CS groups differed reliably on aversion to vinegar, but not to water. In testing with clear vinegar, the RW/CS amd RV/CS groups, extinguished and tested with clear vinegar, showed vinegar intakes of 7.2 and $6.2 \mathrm{~g}$, respectively $(t<1.0)$. The finding that there was no reliable difference in vinegar intakes indicated that, for the RV group,

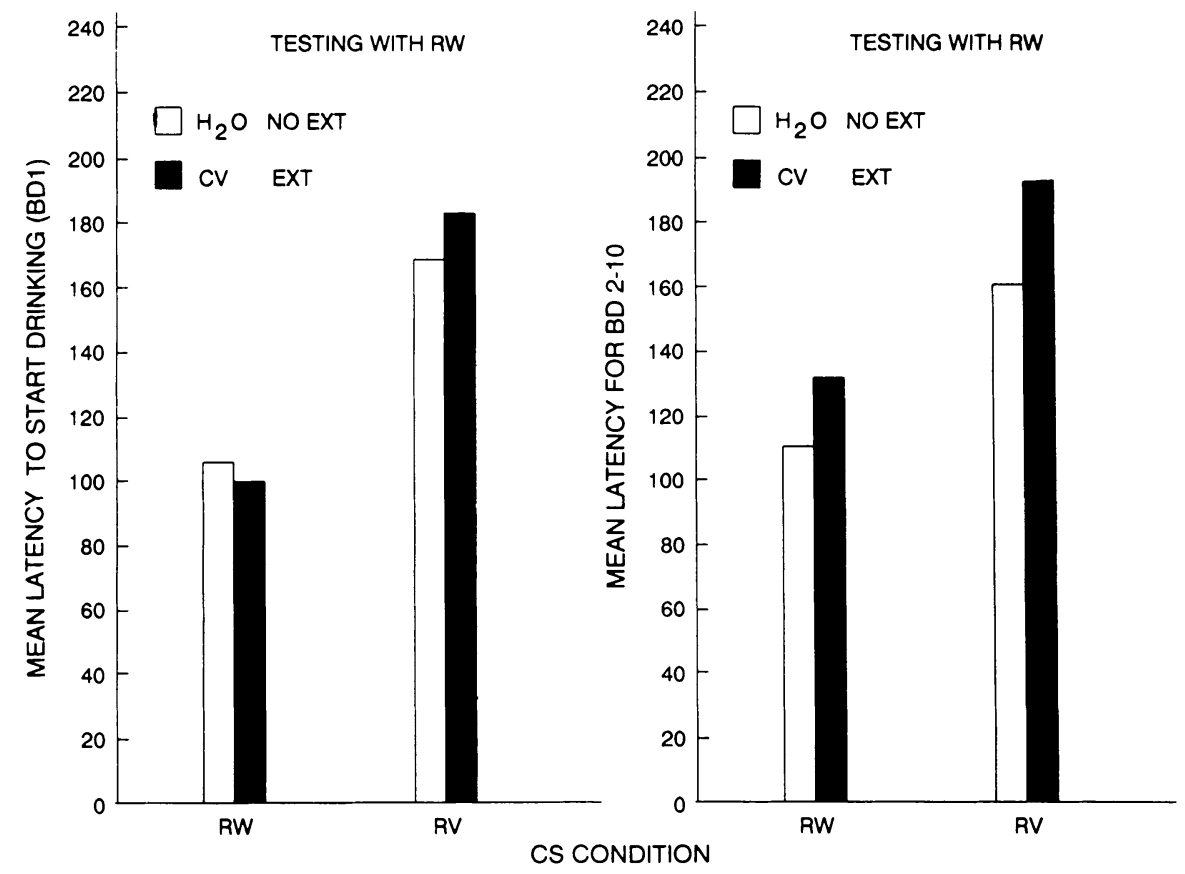

Figure 1. Mean latency (in seconds) to start drinking red water (left panel) and to complete BD 2-10 (right panel) for groups conditioned with red water (RW) or red vinegar (RV) and given access to clear water $\left(\mathrm{H}_{2} \mathrm{O}\right)$ no-extinction or clear vinegar $(\mathrm{CV})$ extinction procedures. 
the extinction procedures reduced the aversion to vinegar to the level of the RW group, which had not been conditioned to vinegar.

\section{DISCUSSION}

Latencies to start drinking reliably showed that taste-mediated potentiation to visual cues survived extinction procedures, which effectively reduced taste aversion to the level of a group that did not have previous taste-aversion conditioning. Latencies to BD 2-10 and intake performance corroborated these results.

These findings support Lett (1984) and contradict predictions from the WCA view (Durlach \& Rescorla, 1980); by implication, they support the SC view. According to that hypothesis, taste cues enhance the associability between visual cues of the compound CS and toxicosis. The fate of taste aversion after the conditioning trial is irrelevant. Postconditioning extinction of taste aversion should not affect the display of potentiation to visual cues; the present data confirmed that expectation.

Since latency to start drinking antedates ingestion, we were able to separate the role of visual cues from that of taste in the display of potentiation. An intake measure permits a confounding between the effects of vision and taste, because intake reflects the role of visual cues in the elicitation of approach behavior and the start of ingestion, and the role of taste cues in the maintenance of ingestion. Further, intake performance may compromise the behavior of potentiation groups and, thus, the size of potentiation. Detection of the familiar taste of water after the start of ingestion in testing may attenuate the display of aversion to visual cues (Lett, 1980, p. 198). On the other hand, gustatory stimulation from drinking in the presence of visual cues may reinstate cues from original conditioning and enhance the magnitude of potentiation. Thus, the presence of taste cues in testing may mitigate the display of aversion to visual cues. The use of a latency measure to determine onset of drinking precluded these potentially contaminating aspects of an intake measure.

The present data also show that relatively brief presentation of the compound CS-four bill dips that provided $.5-.8 \mathrm{~g}$ of intake-produced reliable evidence of potentiation. Other reseachers (e.g., Davis, Best, \& Grover, 1988; Durlach \& Rescorla, 1980; Lett, 1980, 1984; Westbrook, Homewood, Horn, \& Clarke, 1983) typically provided 10-15 min of access to a compound CS on the conditioning day. The present data suggest that chicks rapidly encode visual and taste cues of ingesta on the conditioning trial. The resultant associative structures form, even with the occurrence of the UCS/toxin (Durlach \& Rescorla, 1980). Consequently, the present data agree with those of Lett $(1980,1984)$ and Davis et al. (1988), who determined that preexposure to the compound CS before conditioning may not be a prerequisite for the display of potentiation. The present findings of taste-mediated potentiation to visual cues after relatively brief CS presentation and taste-extinction procedures seem more compatible with a view that the compound CS is a perceptually integrated unit (e.g., Bouton, Dunlap, \& Swartzentruber, 1987; Lett, 1984) rather than an additive combination of elemental stimuli.

\section{REFERENCES}

Bouton, M. E., Dunlap, C. M., \& Swartzentruber, D. (1987). Potentiation of taste by another taste during compound aversion learning. Animal Learning \& Behavior, 15, 433-438.

Bretr, L. P., Hankins, W. G., \& Garcia, J. (1976). Prey-lithium aversions III: Buteo hawks. Behavioral Biology, 17, 87-98.

DAvis, S. F., Best, M. R., \& Grover, C. A. (1988). Toxicosis-mediated potentiation in a taste/taste compound: Evidence for within-compound associations. Learning \& Motivation, 19, 183-205.

Durlach, P. J., \& Rescorla, R. A. (1980). Potentiation rather than overshadowing in flavor-aversion learning: An analysis in terms of within-compound associations. Journal of Experimental Psychology: Animal Behavior Processes, 6, 175-187.

LETT, B. T. (1980). Taste potentiates color-sickness associations in pigeons and quail. Animal Learning \& Behavior, 8, 193-198.

LETT, B. T. (1984). Extinction of taste aversion does not eliminate taste potentiation of odor aversion in rats or color aversion in pigeons. Animal Learning \& Behavior, 12, 414-420.

Rusiniak, K. W., Hankins, W. G., Garcia, J., \& Brett, L. P. (1979). Flavor-illness aversions: Potentiation of odor by taste in rats. $B e-$ havioral \& Neural Biology, 25, 1-17.

Westbrook, R. F., Homewood, J., HorN, K., \& Clarke, J. E. (1983). Flavour-odour compound conditioning: Odour-potentiation and flavour-attenuation. Quarterly Journal of Experimental Psychology, 35B, 13-33.

(Manuscript received May 2, 1993.) 